\title{
OPERATORS WITH DISCONNECTED SPECTRA ARE DENSE
}

\author{
BY DOMINGO A. HERRERO ${ }^{1}$ AND NORBERTO SALINAS ${ }^{2}$
}

Communicated by Fred Gehring, December 13, 1971

\begin{abstract}
It is proven that the set of all (bounded linear) operators on a complex infinite dimensional Banach space having disconnected spectra is an open uniformly dense subset of the algebra of all operators.
\end{abstract}

In [3, Problem 8], P. R. Halmos asked whether the set of all reducible operators in a complex infinite dimensional separable Hilbert space $\mathscr{H}$ is uniformly dense in the algebra $\mathscr{L}(\mathscr{H})$ of all (bounded linear) operators on $\mathscr{H}$. In the present note we answer affirmatively a related question:

Is the set of all operators on a Banach space $X$ having nontrivial complementary hyperinvariant subspaces dense in $\mathscr{L}(\mathscr{X})$ ? (Recall that a subspace $\mathscr{M}$ of $\mathscr{X}$ is hyperinvariant for $T \in \mathscr{L}(\mathscr{X})$ if $A \mathscr{M} \subset \mathscr{M}$, for all $A \in \mathscr{L}(\mathscr{X})$ commuting with $T[1]$. Here and in what follows, subspace means closed linear manifold.)

Moreover, we proved the following stronger (see [4]) result:

THEOREM. Let $\mathscr{X}$ be a complex infinite dimensional Banach space and let $T \in \mathscr{L}(\mathscr{X})$. Then, given any $\varepsilon>0$, there exists an $A \in \mathscr{L}(\mathscr{X})$ such that (1) $\operatorname{rank}(A)=1 ;(2)\|A\|<\varepsilon$, and (3) the spectrum of $T+A$ is disconnected.

Proof. Let $\sigma(T)(E(T)$, resp.) denote the spectrum (essential spectrum, resp.) of $T$.

Let $\lambda_{0}$ be any point of $E(T)$ such that $\operatorname{Re} \lambda_{0}=\max \{\operatorname{Re} \lambda ; \lambda \in E(T)\}$. Then, for every compact operator $K, \lambda_{0} \in E(T+K)=E(T) \subset \sigma(T+K)$, and it follows from [4, Theorem 1] that, if there exists a $\lambda \in \sigma(T+K)$ such that $\operatorname{Re} \lambda>\operatorname{Re} \lambda_{0}$, then $\sigma(T+K)$ is disconnected, $\lambda$ is an isolated point of $\sigma(T+K)$ such that $(T+K-\lambda)^{n} \mathscr{X}$ is closed for every $n \geqq 0$ and, if $\mathscr{M}=\bigcap_{n=1}^{\infty}(T+K-\lambda)^{n} \mathscr{X}$ and $\mathscr{N}=\operatorname{closure}\left\{\bigcup_{n=1}^{\infty} \operatorname{ker}(T+K-\lambda)^{n}\right\}$, then $\operatorname{dim} \mathscr{N}=\operatorname{dim}(\mathscr{X} / \mathscr{M})<\infty$.

Therefore, to complete the proof, it suffices to find an $A$ satisfying (1), (2) and such that $\lambda_{0}+\gamma \in \sigma(T+A)$ for some $\gamma, 0<\gamma<\varepsilon / 2$.

Since $\lambda_{0} \in$ bdry $\sigma(T)$, there exists an $x \in \mathscr{X}$ such that $\|x\|=1$ and $\left\|\left(T-\lambda_{0}\right) x\right\|<\varepsilon / 2$ (see [2, Chapter 7]). By Hahn-Banach theorem, there

AMS 1970 subject classifications. Primary 47A15; Secondary 47A10, 47A55.

Key words and phrases. Complementary subspaces, hyperinvariant subspaces, disconnected spectrum, essential spectrum.

${ }^{1}$ State University of New York at Albany, Albany, New York 12203. Research supported by NSF Grant GU3171.

2 Institute for Advanced Study, Princeton, New Jersey 08540. Research supported in part by NSF Grant GP 7952X3. 
exists a continuous linear functional $f$ on $\mathscr{X}$ such that $f(x)=\|f\|=1$. Define $P \in \mathscr{L}(\mathscr{X})$ by $P y=f(y) x$; then $\|P\|=1$. If $y \in \mathscr{X}$ is a unit vector, then $y$ can be written as $y=\alpha x+z$, where $\alpha$ is a complex number, $|\alpha| \leqq 1$, and $z \in \operatorname{ker}(f)=\operatorname{ker}(P)$.

For each $\gamma, 0<\gamma<\varepsilon / 2$, define $T_{\gamma} \in \mathscr{L}(\mathscr{X})$ by $T_{\gamma}=T(I-P)+\left(\lambda_{0}+\gamma\right) P$; then

$$
\begin{aligned}
\left(T_{\gamma}-T\right) y & =\left[T(I-P)+\left(\lambda_{0}+\gamma\right) P-T\right] y=\left(\lambda_{0}+\gamma-T\right) P y \\
& =\alpha\left(\lambda_{0}+\gamma-T\right) x .
\end{aligned}
$$

Hence $A_{\gamma}=T_{\gamma}-T=\left(\lambda_{0}+\gamma-T\right) P$ has rank one and

$$
\left\|A_{\gamma}\right\|=\sup \left\{\left\|\left(T_{\gamma}-T\right) y\right\|:\|y\|=1\right\}<\varepsilon / 2+\varepsilon / 2=\varepsilon .
$$

Clearly, $\lambda_{0}+\gamma$ is an eigenvalue of $T_{\gamma}$ and therefore $\lambda_{0}+\gamma \in \sigma\left(T_{\gamma}\right)$. The proof is complete.

REMARK. If $\mathscr{M}$ and $\mathcal{N}$ are defined as above (for $\lambda=\lambda_{0}+\gamma$ and $T_{\gamma}$ $=T+A_{y}$ ), then $\mathscr{M}, \mathscr{N}$ are hyperinvariant subspaces of $T$ such that $\mathscr{X}=\mathscr{M} \oplus \mathscr{N}$; moreover, if $\gamma$ is small enough, then $\operatorname{dim} \mathscr{N}=\operatorname{dim} \mathscr{X} / \mathscr{M}$ $=1$. With minor modifications of the same argument it is possible to show that, given $T \in \mathscr{L}(\mathscr{C})$ and $\varepsilon>0$, there exists a compact operator $K$ such that $\|K\|<\varepsilon$ and $\sigma(T+K)$ contains a sequence $\left\{\lambda_{k}: k=1,2, \ldots\right\}$ of isolated eigenvalues associated with hyperinvariant subspaces $\mathscr{N}_{k}, \mathscr{M}_{k}$ (defined as above) such that $\mathscr{X}=\mathscr{M}_{k} \oplus \mathscr{N}_{k}$ and $\operatorname{dim} \mathscr{N}_{k}=\operatorname{dim}\left(\mathscr{X} / \mathscr{M}_{k}\right)$ $=1$, for all $k$. From these results and [4, Theorem 3], we obtain the following:

COROLlary. (1) The set of all $T \in \mathscr{L}(\mathscr{X})$ such that $\sigma(T)$ is disconnected is a uniformly dense open subset of $\mathscr{L}(\mathscr{X})$.

(2) The set of all $T \in \mathscr{L}(\mathscr{X})$ such that, for each $n(n=1,2,3, \ldots), T$ has complementary hyperinvariant subspaces $\mathscr{N}_{n}, \mathscr{M}_{n}$ satisfying

is dense in $\mathscr{L}(\mathscr{X})$.

$$
\operatorname{dim} \mathscr{N}_{n}=\operatorname{dim}\left(\mathscr{X} / \mathscr{M}_{n}\right)=n,
$$

\section{REFERENCES}

1. R. G. Douglas and C. Pearcy, On a topology for invariant subspaces, J. Functional Analysis 2 (1968), 323-341. MR 38 \# 1547.

2. N. Dunford and J. T. Schwartz, Linear operators. I: General theory, Pure and Appl. Math., vol. 7, Interscience, New York, 1958. MR 22 \#8302.

3. P. R. Halmos, Ten problems in Hilbert space, Bull. Amer. Math. Soc. 76 (1970), 887-933. MR 42 \# 5066.

4. N. Salinas, Operators with essentially disconnected spectrum, Acta Sci. Math. (Szeged) (to appear).

Department of Mathematics, State University of New York at Albany, Albany, NEW YORK 12203

InStitute for Advanced Study, Princeton, New Jersey 08540

Current address (Domingo A. Herrero): Universidad Nacional del Sur-Dep. de Matemáticas, Bahía Blanca-Pcia. de Buenos Aires, Argentina. 